\title{
015 PP A LONE VOICE: REFLECTIONS ON PRESENTING QUALITATIVE RESEARCH AT A SURGEON'S CONFERENCE
}

D Trusson. University of Nottingham, UK

\subsection{6/bmjopen-2015-UCLSymposiumAbstracts.26}

In this paper I describe how I used my dual position of service user and sociologist to present my research findings at an international conference for surgeons. As a former breast cancer patient I was invited to participate in a study of women's experiences of having breast reconstruction using their own body tissue. Questions included rating my happiness and confidence on a scale of 1-10 which challenged me as a qualitative researcher. The study also revealed parallels with my own research with 24 women who had been treated for early stage breast cancer, where similar issues had emerged organically through their narratives. When I discussed this with the breast care team I was encouraged to submit an abstract to the conference where the results of the quantitative study were going to be presented.

The subsequent paper, entitled 'Autologous Breast Reconstruction from the Patient's Perspective: A Qualitative Study,' was presented at the $5^{\text {th }}$ International Meeting of Oncoplastic and Reconstructive Breast Surgery in Nottingham in September 2013.

My reflections on my experience of presenting to an audience, who were unaccustomed to qualitative research, include the challenges of facing my own intimidation by health professionals as a patient. I will discuss the positive feedback that I received which will hopefully encourage other qualitative health researchers to reach beyond the usual audiences in order to disseminate their research findings. 